\title{
Long-Term X-ray Findings in Patients With Coronavirus Disease-2019
}

Aarzoo Gupta ${ }^{1}$, Ishan Garg ${ }^{2}$, Abbas Iqbal $^{3}$, Abdul Subhan Talpur ${ }^{4}$, Alyanna Marie B. Mañego ${ }^{5}$, Rama Kalyani Kavuri ${ }^{6}$, Parkash Bachani ${ }^{7}$, Sidra $\mathrm{Naz}^{8}$, Zoya Qamar Iqbal ${ }^{9}$

1. Internal Medicine, Vardhman Mahavir Medical College and Safdarjung Hospital, Faridabad, IND 2. Clinical Medicine, Ross University School of Medicine, Bridgetown, BRB 3. Pediatrics, Ayub Teaching Hospital, Abottabad, PAK 4. Medicine, Liaquat University of Medical and Health Sciences, Jamshoro, PAK 5. Internal Medicine, SeriousMD, Muntinlupa, PHL 6. Internal Medicine, Maratha Vidya Prasarak Samaj's Dr. Vasantrao Pawar Medical College Hospital and Research Centre, Nashik, IND 7. Internal Medicine, Liaquat University of Medical and Health Sciences, Jamshoro, PAK 8. Internal Medicine, University of Health Sciences (UHS), Lahore, PAK 9. Internal Medicine, Jinnah Sindh Medical University, Karachi, PAK

Corresponding author: Sidra Naz, sid8irshad@gmail.com

\section{Abstract}

Introduction: Reverse transcription-polymerase chain reaction (RT-PCR) and chest X-ray (CXR) are commonly used techniques for diagnosing and assessing prognosis in patients with coronavirus disease2019 (COVID-19). This study aims to highlight the long-term radiological findings observed on CXR after recovery, in patients with COVID-19. This will help identify patients suffering from long-term consequences of COVID-19 and help them provide adequate care.

Methods: This study was conducted in the COVID-19 unit of a tertiary care hospital, Pakistan from August 2020 to February 2021. CXR of patients who were being discharged after negative PCR was done. Participants with positive X-ray findings, which included consolidation, reticular thickening, ground-glass opacities (GGO), pulmonary nodules, and pleural effusions, were enrolled in the study after getting informed consent. All findings were recorded in a self-structured questionnaire. Participants were scheduled to come for follow-up on day 30 after their initial CXR, where their CXR was repeated.

Result: Our results showed that $n=429$ (60.2\%) participants had positive CXR at the time of discharge. After 30 days, $n=371$ participants returned for a follow-up X-ray. Out of the 371 participants, after 30 days, 123 participants still had positive CXR. Fatigue (41.4\%) was the common symptom after 30 days. The most common finding was consolidation (82.1\%), followed by reticular thickening (23.5\%) on day 30 .

Conclusion: In this study, although most of the patients completely recovered serologically from COVID-19, they still had radiological findings in their chest X-rays. Radiological findings are especially important in predicting the clinical course of the disease and may be used to monitor long-term complications.

Review began 05/15/2021 Review ended 05/22/2021 Published 05/28/2021

\section{(c) Copyright 2021}

Gupta et al. This is an open access article distributed under the terms of the Creative Commons Attribution License CC-BY 4.0., which permits unrestricted use, distribution, and reproduction in any medium, provided the original author and source are credited.
Categories: Internal Medicine, Infectious Disease, Pulmonology

Keywords: radiological finding, corona virus, covid-19, chest x-ray, long-term impact

\section{Introduction}

The novel coronavirus, widely known as severe acute respiratory syndrome coronavirus 2 (SARS-CoV-2), has infected over 100 million humans worldwide. The first case of coronavirus disease-2019 (COVID-19) was recognized in Wuhan, China in December 2019 [1]. Due to its highly contagious nature, it quickly spread to other parts of the world, leading to the declaration of a pandemic by the World Health Organization (WHO) in March 2020 [2,3].

The symptoms of COVID-19 have been shown to range from mild fever, cough, headache, and fatigue to a more severe presentation with dyspnea, hemoptysis, and atypical pneumonia [4]. In the most severe cases, COVID-19 can lead to severe acute respiratory syndrome, respiratory failure, multi-organ failure with the resultant high mortality $[4,5]$. The diagnosis is confirmed by reverse transcription-polymerase chain reaction (RT-PCR) of samples obtained from oropharyngeal or nasopharyngeal mucosa and is supported by parenchymal changes seen on chest imaging [6,7]. The more common radiological changes noted on the chest imaging include ground-glass opacities (GGO) and consolidations while cavitation, pleural effusions, pleural thickening, bronchiectasis, and lymphadenopathy occur less frequently [8-10].

Improvement in the chest X-ray (CXR), along with negative PCR, has been seen to guide patient discharge in several studies [11]. Although RT-PCR and CXR are the more commonly used techniques for diagnosis, computed tomography (CT) scans of the chest have shown to have superiority in terms of higher sensitivity (98\%) and can diagnose the disease even prior to symptom onset [12]. For this reason, most studies published in COVID-19 patients describe parenchymal changes seen on CT scans [9,10]. Limited studies 
have highlighted the role of CXR in monitoring disease course and in the follow-up of long-term changes in the lung parenchyma [7,13]. As CXR is the more readily available and cheaper imaging modality, it is essential that its role in disease progression and disease improvement should be known. Therefore, this study aims to highlight the long-term radiological findings observed on CXR after recovery, in patients with COVID-19.

\section{Materials And Methods}

This study was conducted in the COVID-19 unit of a tertiary care hospital, Pakistan from August 2020 to February 2021. CXR of patients who were being discharged after recovery was done and participants with positive X-ray findings were enrolled in the study after informed consent via consecutive convenient nonprobability sampling. Recovery was defined as a negative PCR test for COVID-19. Positive CXR findings were consolidations, reticular thickening, GGO, pulmonary nodules, and pleural effusions. Ethical review board approval was taken.

Out of 712 participants, $n=429$ (60.25\%) participants had positive CXR at the time of discharge. All findings were recorded in a self-structured questionnaire. The highest C-reactive protein (CRP) and lactate dehydrogenase (LDH) were recorded in questionnaires. Participants were scheduled for follow-up on day 30 after their initial chest X-ray, where their CXR was repeated. Fifty-eight (58) participants were lost to followup. Only participants who completed the study were included in the final analysis.

Statistical analysis was done using Statistical Package for the Social Sciences (SPSS) v. 22.0 (IBM Corporation, Armonk, New York, USA). Categorical data were presented as frequency and percentage. Numerical data were presented as mean and standard deviation.

\section{Results}

Out of 712 participants, $n=429$ (60.2\%) participants had positive CXR at the time of discharge. The most common symptom at the time of discharge was fatigue (77.3\%). The most common finding was consolidation (78.9\%), followed by reticular thickening (24.5\%). In terms of location of findings, $66.5 \%$ of participants had bilateral radiological findings (Table 1 ). 


\section{Cureus}

\section{Characteristics}

Age in years (mean $\pm S D)$

Gender

Male

Female

Laboratory findings

CRP (mg/L)

LDH (IU)

Symptom at discharge

Fatigue

Cough

Dyspnea

SOB

Chest X-ray findings (location)

Right

Left

Both

Radiological findings

Consolidation

Reticular thickening

GGO

Pulmonary nodules

Pleural effusions

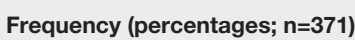

$42 \pm 10$

$179(48.2 \%)$

$192(51.8 \%)$

$122.7 \pm 21.4$

$328.1 \pm 97.1$

$287(77.3 \%)$

$101(27.2 \%)$

$61(16.4 \%)$

$58(15.6 \%)$

$62(16.7 \%)$

$65(17.5 \%)$

$247(66.5 \%)$

$293(78.9 \%)$

$91(24.5 \%)$

$81(21.8 \%)$

$20(5.3 \%)$

$18(4.8 \%)$

\section{TABLE 1: Symptoms, laboratory, and radiological findings on discharge.}

CRP: C-reactive protein, GGO: ground-glass opacities, LDH: lactate dehydrogenase, SOB: shortness of breath, mg/L: milligram per liter, IU: International Unit.

After 30 days, 371 participants returned for a follow-up X-ray. Out of 371 participants, on day 30, 123 (33.1\%) participants still had positive CXR; 51 (41.4\%) participants reported fatigue; 63.4\% had bilateral radiological findings and the most common finding was consolidation $(82.1 \%)$, followed by reticular thickening (23.5\%; Table 2). 


\section{Cureus}

\begin{tabular}{|c|c|}
\hline Characteristics & Frequency (percentages; $n=123$ ) \\
\hline \multicolumn{2}{|l|}{ Gender } \\
\hline Male & $63(51.3 \%)$ \\
\hline Female & 60 (48.7\%) \\
\hline \multicolumn{2}{|l|}{ Symptom at 30 days } \\
\hline Fatigue & $51(41.4 \%)$ \\
\hline Cough & $22(17.8 \%)$ \\
\hline SOB & $15(12.1 \%)$ \\
\hline Dyspnea & $18(8.1 \%)$ \\
\hline \multicolumn{2}{|c|}{ Chest $\mathrm{X}$-ray findings at 30 days (location) } \\
\hline Right & $22(17.8 \%)$ \\
\hline Left & $23(18.6 \%)$ \\
\hline Both & $78(63.4 \%)$ \\
\hline \multicolumn{2}{|l|}{ Radiological findings } \\
\hline Consolidation & $101(82.1 \%)$ \\
\hline Reticular thickening & $29(23.5 \%)$ \\
\hline GGO & $20(16.2 \%)$ \\
\hline Pleural effusions & $06(4.8 \%)$ \\
\hline Pulmonary nodules & $05(4.1 \%)$ \\
\hline
\end{tabular}

\section{TABLE 2: Symptoms and radiological findings on 30-day follow-up}

GGO: ground-glass opacities, SOB: shortness of breath.

\section{Discussion}

The result of our study demonstrated that 429 (60.9\%) participants had positive CXR at the time of discharge. Among the symptoms, fatigue (77\%) was found to be the most common one at the time of discharge. In terms of location of findings, $66.5 \%$ of participants had bilateral radiological findings. Consolidation (78.9\%), followed by reticular thickening (24.5\%) was the most common finding. 371 patients returned for a follow-up after 30 days, of which 123 (33.1\%) participants still had positive CXR. After 30 days, $41.4 \%$ of the patients reported symptoms of fatigue, $63.4 \%$ had bilateral radiological findings and the most common finding was consolidation (82.1\%), followed by reticular thickening (23.5\%).

The results of our study are in line with other studies $[2,14]$. In concordance with our study, results from the systematic review of imaging findings conducted by Salehi et al. also showed the initial finding of CT scan in COVID-19 patients is bilateral, multilobar GGO with a peripheral or posterior distribution (or both), mainly in the lower lobes and less frequently within the right middle lobe [2]. Some studies also suggested that CT findings may vary among age groups, with consolidation more common in older groups and GGO in younger people [14]. The CT findings of COVID-19 patients in Korea were similar to those in China. However, the proportion of consolidation lesions were more common in Chinese people. On the basis of these radiological findings, Korean patients seem to experience a milder disease course than Chinese people [15]. In cases of long-term follow-up, studies have shown that approximately $98.1 \%$ of the chest CTs will show abnormalities even after 28 days of the onset of symptoms [16], and several case reports have demonstrated that postdischarge chest CTs show abnormalities but with slight improvements [17,18]. This has also been demonstrated in the findings of our study.

Even though the clinical and radiological course of COVID-19 is not predictable [19,20], there is proof that complications can exist for over a month. Even if patients do not show symptoms after being discharged for quite a few weeks or months, the idea of reinfection cannot go unnoticed. Additionally, abnormalities in the pulmonary function and radiological findings will exist and should be monitored for a long span of time. These could be more prominent in patients with pre-existing pulmonary or extrapulmonary diseases [21-23]. 
To the best of our knowledge, it is the first study from this region that highlights the long-term impact of COVID-19 on chest X-ray findings. However, since the study was conducted in a single institute, care should be taken while implying its result for a large population. Further large-scale studies are needed to identify the long-term CXR findings associated with COVID-19.

\section{Conclusions}

In the case of COVID-19, keeping the long-term abnormal CXR in mind, follow-up visits after discharge are particularly important. They are just a check-up to observe changes in patient's clinical conditions and ensure they are not any complications. It is also a great time to talk to a primary care provider about anything else or ask questions about mental wellbeing. The goal is to keep patients healthy and out of the hospital to prevent complications from happening again. Although most of the patients completely recovered from the disease, they are likely to have long-term lung damage. Radiological findings are particularly important in predicting the clinical course of the disease and may be used to monitor long-term complications.

\section{Additional Information \\ Disclosures}

Human subjects: Consent was obtained or waived by all participants in this study. University of Health Sciences Lahore issued approval UHS/IRB-Ofc/2020/21. Animal subjects: All authors have confirmed that this study did not involve animal subjects or tissue. Conflicts of interest: In compliance with the ICMJE uniform disclosure form, all authors declare the following: Payment/services info: All authors have declared that no financial support was received from any organization for the submitted work. Financial relationships: All authors have declared that they have no financial relationships at present or within the previous three years with any organizations that might have an interest in the submitted work. Other relationships: All authors have declared that there are no other relationships or activities that could appear to have influenced the submitted work.

\section{References}

1. Chakraborty C, Sharma AR, Sharma G, Bhattacharya M, Lee SS: SARS-CoV-2 causing pneumonia-associated respiratory disorder (COVID-19): diagnostic and proposed therapeutic options. Eur Rev Med Pharmacol Sci. 2020, 24:4016-2. 10.26355/eurrev_202004_20871

2. Salehi S, Abedi A, Balakrishnan S, Gholamrezanezhad A: Coronavirus disease 2019 (COVID-19): a systematic review of imaging findings in 919 patients. AJR Am J Roentgenol. 2020, 215:87-93. 10.2214/AJR.20.23034

3. Chan JF, Yip CC, To KK, et al.: Improved molecular diagnosis of COVID-19 by the novel, highly sensitive and specific COVID-19-RdRp/Hel real-time reverse transcription-PCR assay validated In vitro and with clinical specimens. J Clin Microbiol. 2020, 58:e00310-20. 10.1128/JCM.00310-20

4. Chen J, Qi T, Liu L, et al.: Clinical progression of patients with COVID-19 in Shanghai, China . J Infect. 2020, 80:e1-6. 10.1016/j.jinf.2020.03.004

5. Huang C, Wang Y, Li X, et al.: Clinical features of patients infected with 2019 novel coronavirus in Wuhan, China. Lancet. 2020, 395:497-506. 10.1016/S0140-6736(20)30183-5

6. Ai T, Yang Z, Hou H, et al.: Correlation of chest CT and RT-PCR testing for Coronavirus disease 2019 (COVID-19) in China: a report of 1014 cases. Radiology. 2020, 296:E32-40. 10.1148/radiol.2020200642

7. Lei Y, Zhang HW, Yu J, Patlas MN: COVID-19 infection: early lessons. Can Assoc Radiol J. 2020, 71:251-2. $10.1177 / 0846537120914428$

8. Yoon SH, Lee KH, Kim JY, et al.: Chest radiographic and CT findings of the 2019 novel Coronavirus disease (COVID-19): analysis of nine patients treated in Korea. Korean J Radiol. 2020, 21:494-500. 10.3348/kjr.2020.0132

9. Caruso D, Zerunian M, Polici M, et al.: Chest CT features of COVID-19 in Rome, Italy . Radiology. 2020, 296:E79-85. 10.1148/radiol.2020201237

10. Bernheim A, Mei X, Huang M, et al.: Chest CT findings in Coronavirus disease-19 (COVID-19): relationship to duration of infection. Radiology. 2020, 295:200463. 10.1148/radiol.2020200463

11. Zheng Z, Yao Z, Wu K, Zheng J: Patient follow-up after discharge after COVID-19 pneumonia: considerations for infectious control. J Med Virol. 2020, 92:2412-9. 10.1002/jmv.25994

12. Fang Y, Zhang H, Xie J, Lin M, Ying L, Pang P, Ji W: Sensitivity of chest CT for COVID-19: comparison to RT-PCR. Radiology. 2020, 296:E115-7. 10.1148/radiol.2020200432

13. Zhang J, Tian S, Lou J, Chen Y: Familial cluster of COVID-19 infection from an asymptomatic . Crit Care. 2020, 24:119. 10.1186/s13054-020-2817-7

14. Kanne JP: Chest CT findings in 2019 novel Coronavirus (2019-nCoV) infections from Wuhan, China: key points for the radiologist. Radiology. 2020, 295:16-7. 10.1148/radiol.2020200241

15. Ng MY, Lee EYP, Yang J, et al.: Imaging profile of the COVID-19 infection: radiologic findings and literature review. Radiol Cardiothorac Imaging. 2020, 2:e200034. 10.1148/ryct.2020200034

16. Ding X, Xu J, Zhou J, Long Q: Chest CT findings of COVID-19 pneumonia by duration of symptoms . Eur J Radiol. 2020, 127:109009. 10.1016/j.ejrad.2020.109009

17. Dou P, Zhang S, Wang C, et al.: Serial CT features in discharged COVID-19 patients with positive RT-PCR re-test. Eur J Radiol. 2020, 127:109010. 10.1016/j.ejrad.2020.109010

18. Fu W, Chen $\mathrm{Q}$, Wang T: Three cases of re-detectable positive SARS-CoV-2 RNA in recovered COVID-19 patients with antibodies. J Med Virol. 2020, 92:2298-301. 10.1002/jmv.25968

19. Sheehy LM: Considerations for postacute rehabilitation for survivors of COVID-19 . JMIR Public Health Surveill. 2020, 6:e19462. 10.2196/19462 


\section{Cureus}

20. Kooraki S, Hosseiny M, Myers L, Gholamrezanezhad A: Coronavirus (COVID-19) outbreak: what the department of radiology should know. J Am Coll Radiol. 2020, 17:447-51. 10.1016/j.jacr.2020.02.008

21. Hosseiny M, Kooraki S, Gholamrezanezhad A, Reddy S, Myers L: Radiology perspective of Coronavirus disease 2019 (COVID-19): lessons from severe acute respiratory syndrome and middle east respiratory syndrome. AJR Am J Roentgenol. 2020, 214:1078-82. 10.2214/AJR.20.22969

22. Behzad S, Aghaghazvini L, Radmard AR, Gholamrezanezhad A: Extrapulmonary manifestations of COVID19: radiologic and clinical overview. Clin Imaging. 2020, 66:35-41. 10.1016/j.clinimag.2020.05.013

23. Katal S, Aghaghazvini L, Gholamrezanezhad A: Chest-CT findings of COVID-19 in patients with preexisting malignancies: a pictorial review. Clin Imaging. 2020, 67:121-9. 10.1016/j.clinimag.2020.06.004 